\title{
Effect of alendronate on resorption of transplanted bone in mice
}

Toshitsugu Kawata, Kaoru Tenjou, Chiyoko Tokimasa, Tadashi Fujta, Masato Kaku, Akira Matsuki, Shinya Kohno, Keisuke Tsutsui, Junji Ohtani, Masahide Motokawa, Takashi Kobayakawa, Mao Shigekawa, Yuiko TOHMA and Kazuo TANNE

Department of Orthodontics and Craniofacial Developmental Biology, Graduate School of Biomedical Sciences, Hiroshima University, 1-2-3 Kasumi, Minami-ku, Hiroshima 734-8553, Japan

(Accepted $9 \mathrm{Jul}, 2003$ )

\begin{abstract}
Alendronate, one of the bisphosphonates, is known to have an inhibitory effect on bone resorption. The purpose of study was to investigate the effects of alendronate on ectopic bone graft resorption and to determine the optimal dose in the mouse. In control group, segments of chopped tarsal bone were immersed in saline solution and auto-grafted. In experimental groups, the same bone segments were immersed in alendronate ranging in concentration from $10^{-4}$ to $10^{-6} \mathrm{M}$ prior to transplantation. The grafted bone in the control group disappeared due to resorption by osteoclasts within 5 weeks. In the experimental groups, the area of bone tissue decreased by only $20-40 \%$ at 5 weeks post-operatively. The transplanted bone volume in alendronate-treated groups exhibited a tendency of dose-dependent increase, being highest in the $10^{-4} \mathrm{M}$ group. The number of osteoclasts was significantly less in the alendronate-treated groups than in the controls. These results suggested that the alendronate inhibits resorption of ectopic bone graft at a concentration of $10^{-4}$ to $10^{-6} \mathrm{M}$.
\end{abstract}

Regeneration of bone in osseous defects resulting from maxillary alveolar clefts, as well as in craniofacial bone clefts of the palate, is of paramount importance in the restoration of lost form and function. At present, the most reliable bone regenerative material for maxillary alveolar clefts and cleft palate is fresh autogeneous graft, but it requires increased surgical duration and may cause ill effects at the donor site such as excessive blood loss, pneumothorax, wound infection, chronic pain, parpneumothorax, wound infection, paresthesia, unsightly scars, and a potential disturbance of iliac development in the young (6). Bisphosphonates suppress osteoclastic bone resorption and are used to treat bone disorders including metastatic bone disease and osteoporosis (5). Although the molecular mechanism of the action re-

Address correspondence to: Dr. Toshitsugu Kawata at the above address.

Tel: +81-82-257-5686 Fax: +81-82-257-5687

E-mail: tenzan@hiroshima-u.ac.jp mains to be elucidated, it is currently thought that their anti-resorptive action is of suppression in the formation of osteoclasts from hemopoietic precursors that originate in the bone marrow (11). The relative contribution of these mechanisms to the action of bisphosphonates in vivo is not known and may not be the same for every individual compound. In the previous studies, the influence of immersing transplanted bone in bisphosphonates was not considered. This study investigated the effects of alendronate on ectopic bone graft resorption and determined the optimal dose.

C57BL/6J male and female mice were obtained from Jackson Laboratory (Bar Harbor, ME, USA). Newborn male mice were kept in metal cages $(22 \times$ $32 \times 11 \mathrm{~cm})$ with autoclaved wood chips for bedding in an animal room (temperature $24 \pm 2^{\circ} \mathrm{C}$; relative humidity $50 \pm 5 \%$ ). Newborn mice were weaned at 20 days after birth. The study was approved by Animal Use Committee, Hiroshima University, and the animals were maintained in 
accordance with the guidelines issued by Hiroshima University for the care and use of laboratory animals.

In each of the experimental and control groups, three mice weighing about $30 \mathrm{~g}$ each were used. Tarsal bone, $1 \mathrm{mg}$ in weight, was chopped and dipped in saline or alendronate (Banyu Pharmaceutical Co., Tokyo, Japan) solution before autograft. The mice were divided into four groups: $10^{-4} \mathrm{M}$ alendronate immersed group $(\mathrm{n}=3), 10^{-5} \mathrm{M}$ alendronate immersed group $(\mathrm{n}=3), 10^{-6} \mathrm{M}$ alendronate immersed group $(n=3)$ and saline group $(n=3)$. Bone material was grafted into the subcutaneous layer under general anesthesia (Fig. 1). Dorsoventral and lateral $X$-rays of each mouse were taken at $1,2,3,4,5,6$, 7,8 and 9 weeks after grafting. The cephalometric $\mathrm{X}$-ray apparatus for the rat and mouse (Asahi Roentogen Ind. Co., Kyoto, Japan) was used at $20-25 \mathrm{kV}$ and $6 \mathrm{~mA}$ with an exposure time of $3.0 \mathrm{sec}$ for $\mathrm{Ko}-$ dak Dental Ultra-speed film ${ }^{\mathbb{1}}$ (Eastman Kodak Co., Rochester, NY, USA). Transplanted bone volume was analyzed on the radiograph using an imageanalysis program (NIH Image 1.59; National Institutes of Health, Bethesda, MD, USA).

Three mice from each group were sacrificed following anesthetization by an intraperitoneal injection of sodium pentobarbital at 1, 2, 3, 4, 5, 6, 7, 8 and 9 weeks after grafting. The transplanted bone materials were removed, fixed in $4 \%$ paraformaldehyde for $12 \mathrm{~h}$, and then rinsed in distilled water. The specimens were decalcified in 14\% EDTA (pH 7.4) for 14 days at $4^{\circ} \mathrm{C}$, embedded in paraffin, and cut into longitudinal sections of $7 \mu \mathrm{m}$ thickness. These

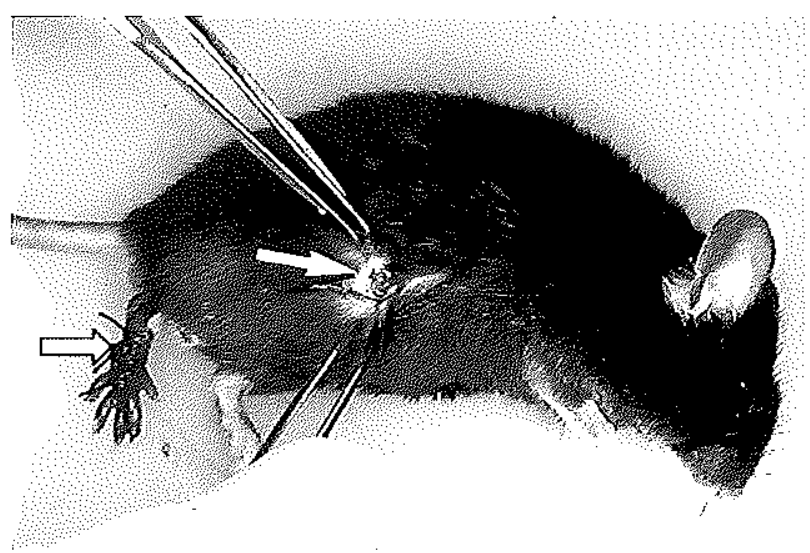

Fig. 1 Bone material was autografted into the subcutaneous layer under general anesthesia. An open arrow indicates tarsal bone fragment. A white arrow indicates the subcutaneous layer where the bone fragment should be transplanted. sections were stained with tartrate-resistant acid phosphatase (TRAP) for the detection of osteoclasts and counterstained with hematoxylin. The number of osteoclasts was counted on each section.

To examine the differences in body weight, the diameter of transplanted bone and the number of osteoclasts, analysis of variance (ANOVA) and Fisher's test were performed using Stat View ${ }^{\mathbb{R}}$ (Abacus Concepts).

There were no significant differences in body weights among the four groups throughout the whole experimental period (Fig. 2). It was thus confirmed that no experimental and surgical invasions exerted any substantial influences on the general growth of the mice.

In the control group, the grafted bone disappeared by resorption by 5 weeks after surgery (Fig. 3). At 2 weeks after surgery, the decreased area of bone structure was significantly less in all the alendronateimmersed groups than in the controls (Figs. 3 and 4). In experimental groups grafted with bones immersed in $10^{-4}$ to $10^{-6} \mathrm{M}$ of alendronate, the area of bone tissue were decreased only by about $20 \% 2$ weeks post-operatively. Thereafter, the area decreased slightly, and maintained the sufficient bone volume until 9 weeks (Fig. 3). The bone volume appeared highest in the $10^{-4} \mathrm{M}$ group showing a tendency of dose-dependent increase, but no significant differences in bone volume were found between groups induced by different doses of bisphosphonate at concentration from $10^{-4} \mathrm{M}$ to $10^{-6} \mathrm{M}$ (Fig. 3). The number of TRAP-positive osteoclasts rapidly increased until 2 weeks after bone transplanting in

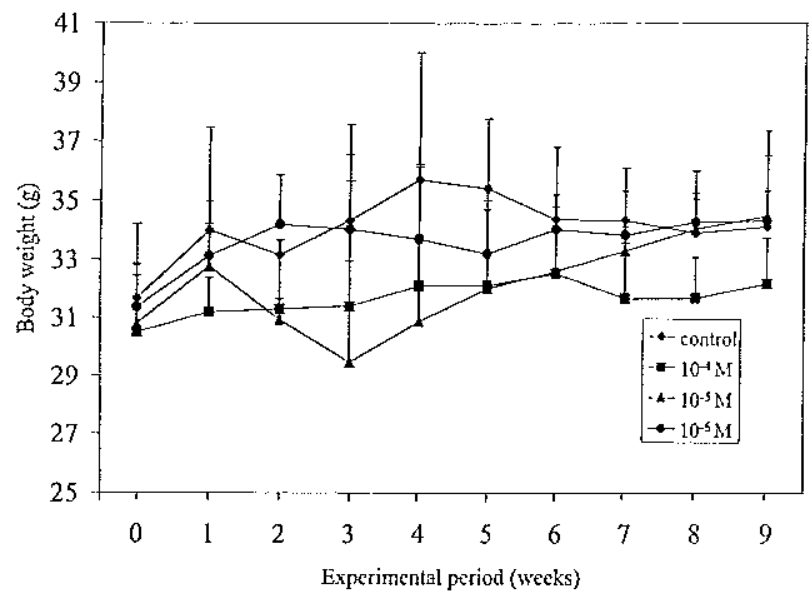

Fig. 2 Changes in body weight for four groups. The receiving bones were immersed in $10^{-4} \mathrm{M}$ alendronate (n =3), $10^{-5} \mathrm{M}$ alendronate $(\mathrm{n}=3), 10^{-6} \mathrm{M}$ alendronate ( $=3$ ) or saline $(n=3)$. 
control group, and then decreased rapidly, due to the loss of bone volume (Fig. 5). The number of osteoclasts in the experimental groups were significantly less than those in the controls until 3 weeks after sur- gery (Figs. 5 and 6). Although these effects seen in all concentration may be enough at clinical application, we have to change alendronate concentration taking the quantity of tissue fluid into consideration.

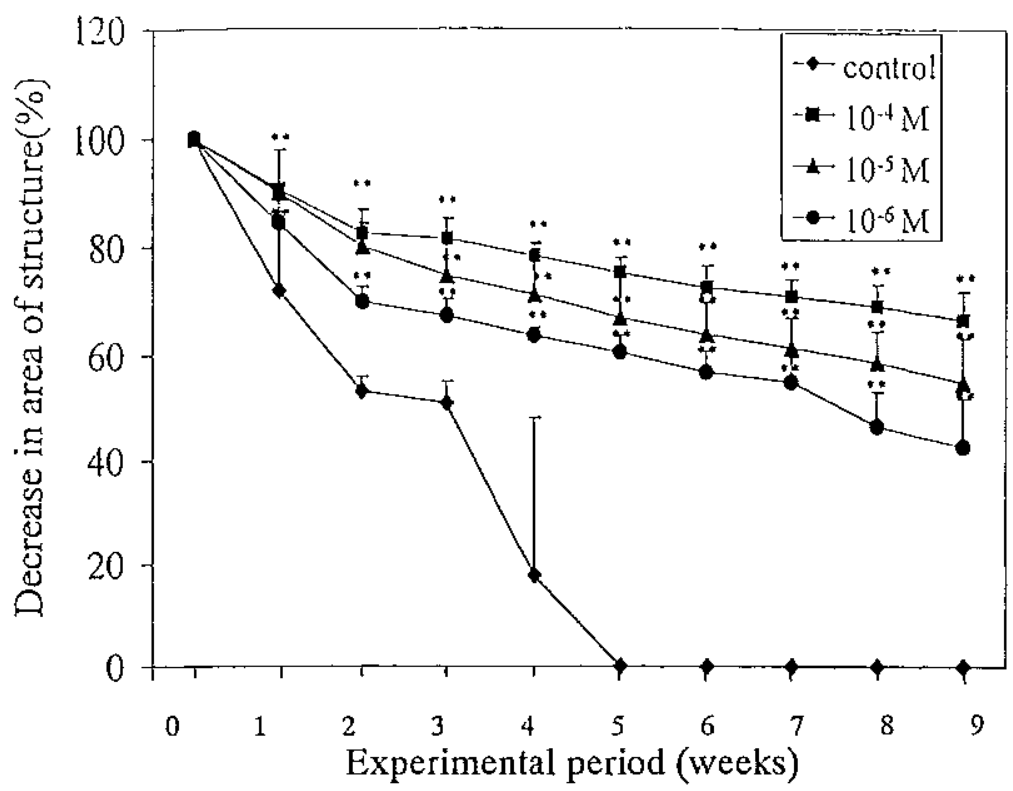

Fig. 3 Changes in the bone volume of transplanted bone, as analysed by dorsoventral and lateral X-rays of each mouse. Significantly different $* *(p<0.01)$ VS control.
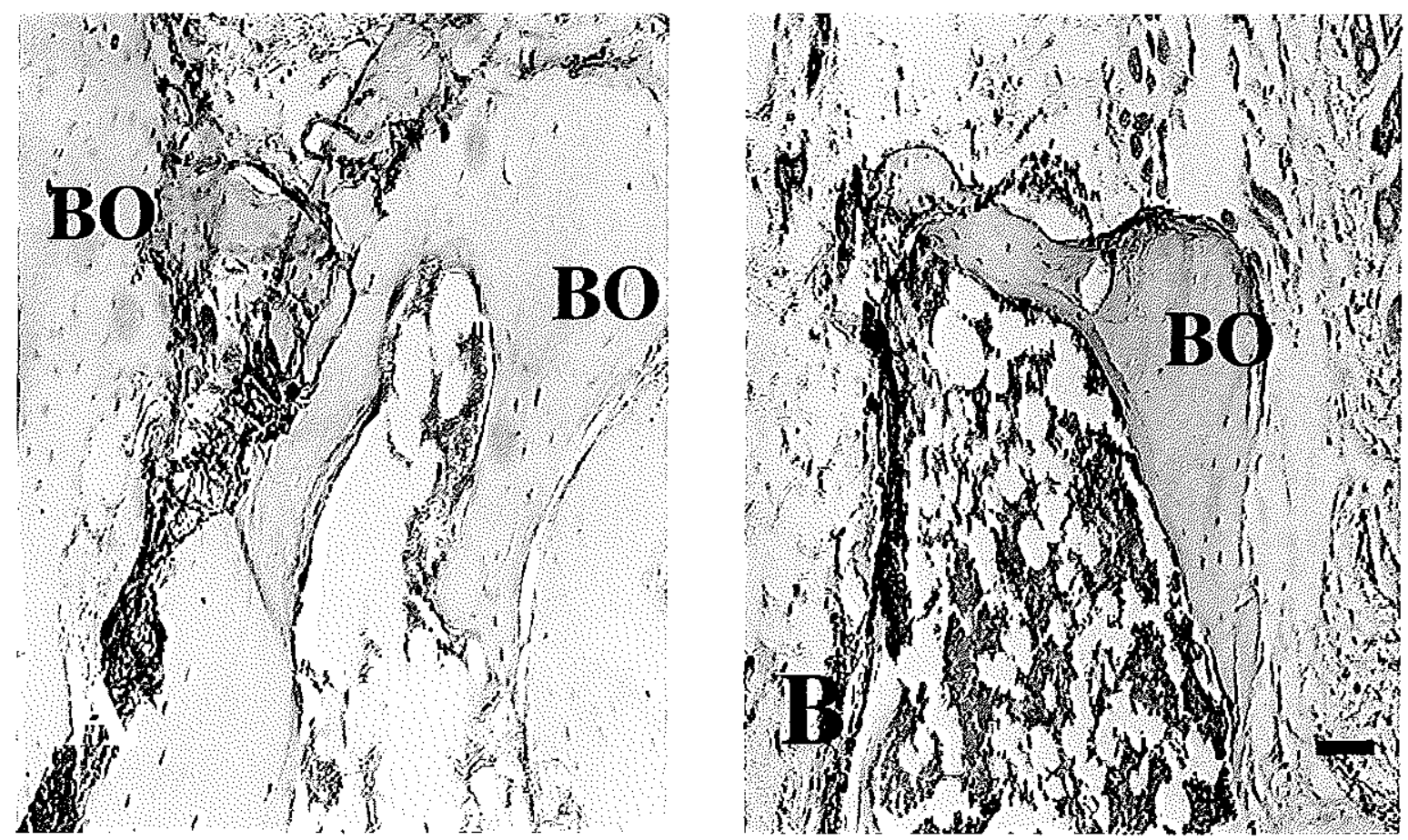

Fig. 4 Microphotographs of transplanted bones (BO) 3 weeks after bone transplantation. Control (A) and $10^{-4} \mathrm{M}$ alendronate-treated mouse (B). (TRAP activity counterstained with hematoxylin, horizontal bar $=50 \mu \mathrm{m}$ ) 
The previous studies clearly demonstrated the induction of osteoclast apoptosis by bisphosphonates $(1,4,7,8,9)$. Furthermore, osteoclast apoptosis may occur as a result of treatment with any of the bisphosphonate family, and certainly reduce bone resorption. Bisphosphonates have no direct effect on calcium transport from the intestine (3) or on renal calcium reabsorption in laboratory animals (10) and

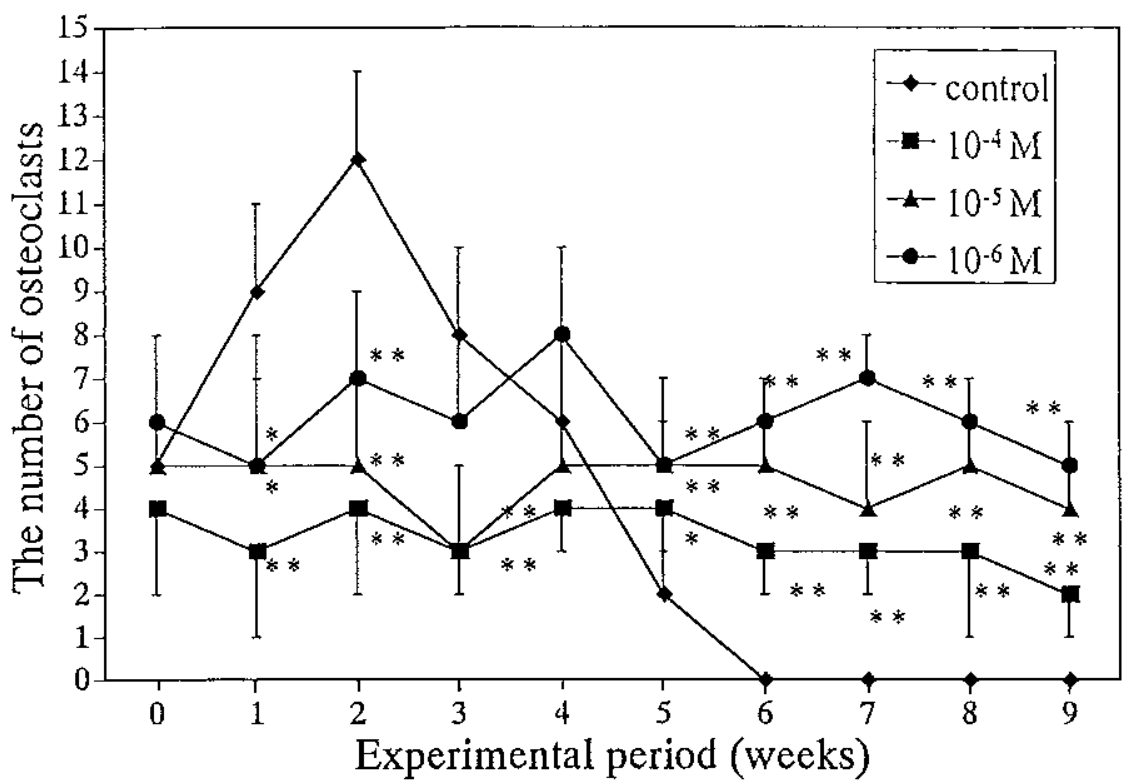

Fig. 5 Changes in the number of osteoclasts detected by TRAP histochemistry. Significantly different $* *(p<0.01)$ and $*(p<0.05)$ VS control.
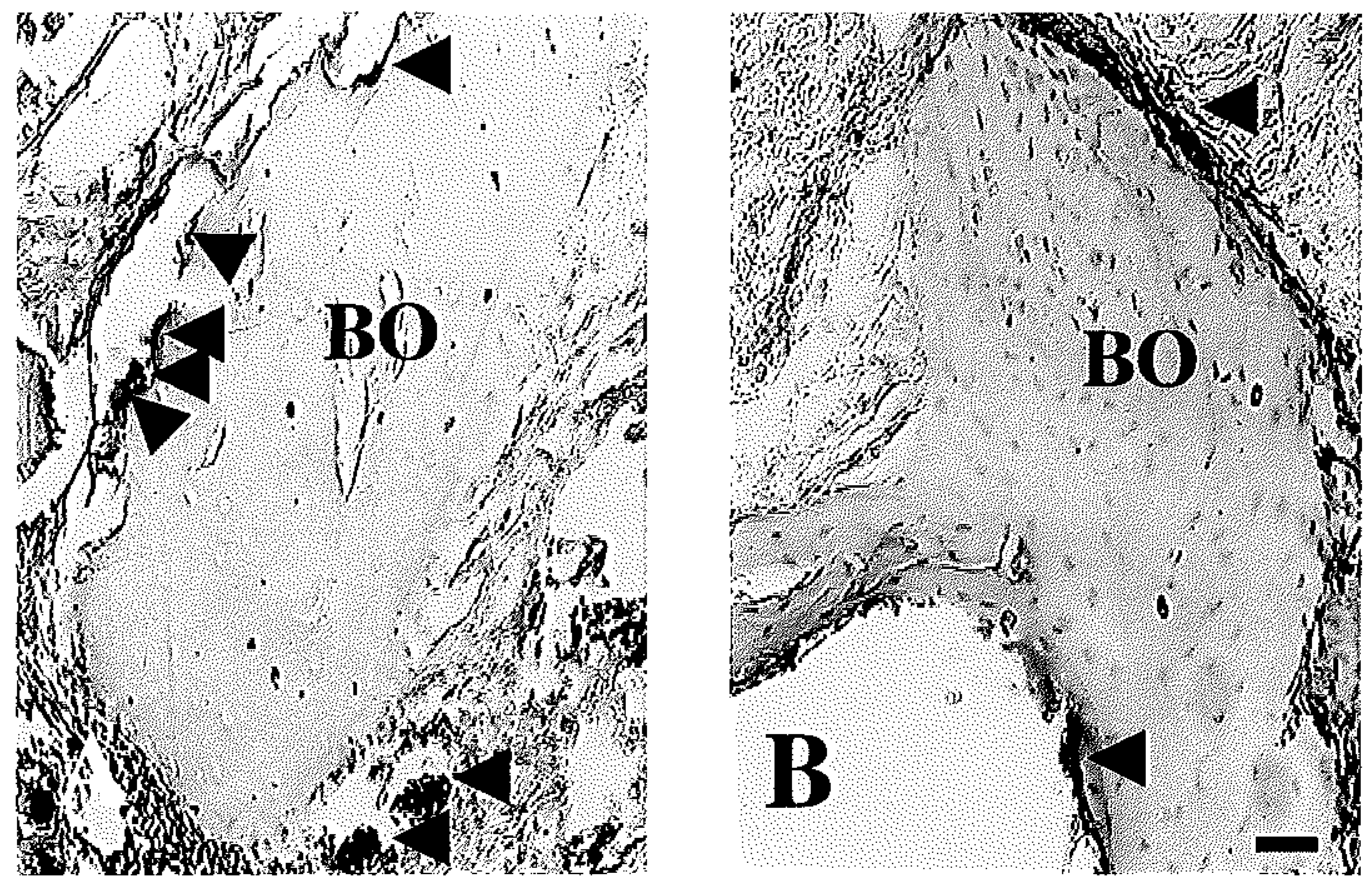

Fig. 6 TRAP-positive cells appeared around the grafted bone (BO) 2 weeks after bone grafting. Control (A) and $10^{-4} \mathrm{M}$ alendronate-treated mouse (B). Arrowheads indicate TRAP-positive cells. (TRAP activity counterstained with hematoxylin, horizontal bar $=50 \mu \mathrm{m}$ ) 
in patients with malignant hypercalcemia (2). Thus, the alendronate may prevent differentiation of osteoclasts and/or induce apoptosis of osteoclasts. The contribution of alendronate to the bone resorption mechanisms may depend on the dose of drug and schedule of administration. The present study suggests that the bone resorption in early stages of bone transplantation is controled by a local action of alendronate.

In conclusion, the transplanted bone volume in alendronate-treated groups was substantially higher than that in non-treated group with a tendency of dose-dependent increase. These results suggested that alendronate inhibits resorption of ectopic bone graft at a concentration of $10^{-4}, 10^{-5}$ and $10^{-6} \mathrm{M}$.

\section{Acknowledgements}

The authors are grateful to the staff of the Research Facility of Laboratory Animal Science, Hiroshima University School of Medicine, for their assistance.

\section{REFERENCES}

1. Benford H. L., Frith J. C., Auriola S., Monkkonen J. and Rogers M. J. (1999) Farnesol and geranylgeraniol prevent activation of caspases by aminobisphosphonates: biochemical evidence for two distinct pharmacological classes of bisphosphonate drugs. Mol. Pharmacol. 56, 131-140.

2. Bickerstaff D. R., O'Doherty D. P., McCloskey E. V., Hamdy N. A., Mian M. and Kanis J. A. (1991) Effects of amino-butylidene diphosphonate in hypercalcemia due to malignancy. Bone 12, 17-20.
3. Bonjour J. P., Pussel R. G. G., Morgan D. B. and Fleisch H. (1973) Intestinal calcium absorption, Ca-binding protein, and Ca-ATPase in diphosphonate-treateed rats. Am. J. Physiol. 224, 1011-1017.

4. Coxon F. P., Helfrich M. H., Van't Hof. R., Sebti S., Ralston S. H., Hamilton A and Rogers M. J. (2000) Protein geranylgeranylation is required for osteoclast formation, function, and survival: inhibition by bisphosphonates and GGTI-298. J. Bone Miner: Res. 15, 1467-1476.

5. Fleisch H. (1991) Bisphosphonates. Pharmacology and use in the treatment of tumour-induced hypercalcaemic and metastatic bone disease. Drugs 42, 919-944.

6. Glowacki J., Kaban L. B., Murray J. E., Folkman J. and Mulliken J. B. (1981) Application of the biological principle of induced osteogenesis for craniofacial defects. Lancet 1,959962.

7. Hughes D. E., Wright K. R., Uy H. L., Sasaki A., Yoneda T., Roodman G. D., Mundy G. R. and Boyce B. F. (1995) Bisphosplionates promote apoptosis in murine osteoclasts in vitro and in vivo. J. Bone Miner: Res. 10, 1478-1487.

8. Luckman S. P., Hughes D. E., Coxon F. P., Graham R., Russell G and Rogers M. J. (1998) Nitrogen-containing bisphosphonates inhibit the mevalonate pathway and prevent posttranslational prenylation of GTP-binding proteins, including Ras. J. Bone Miner: Res. 13, 581-589.

9. Reszka A. A., Halasy-Nagy J. M., Masarachia P. J. and Rodan G. A. (1999) Bisphosphonates act directly on the osteoclast to induce caspase cleavage of mstl kinase during apoptosis. A link between inhibition of the mevalonate pathway and regulation of an apoptosis-promoting kinase. J. Biol. Chem. 274, 34967-34973.

10. Rizzoli R., Caverzasio J., Chapuy M. C., Martin T. J. and Bonjour J. P. (1989) Role of bone and kidney in parathyroid hormone-related peptide-induced hypercalcemia in rats. $J$. Bone Miner: Res. 4, 759-765.

11. Rodan G. A. and Fleisch H. A. (1996) Bisphosphonates: mechanisms of action. J. Clin. Invest. 97, 2692-2696. 